\title{
Magnetic transition in nanocrystalline soft magnetic alloys analyzed via ac inductive techniques
}

\author{
C. Gómez-Polo,* J. I. Pérez-Landazabal, and V. Recarte \\ Dept. de Física, Universidad Pública de Navarra, Campus de Arrosadía, 31006 Pamplona, Spain \\ M. Vázquez \\ Inst. de Ciencia de Materiales, CSIC, Campus de Cantoblanco, 28049 Madrid, Spain \\ A. Hernando \\ Inst. de Magnetismo Aplicado (UCM-RENFE), P.O. Box 155, 28230 Las Rozas, Spain
}

(Received 23 April 2004; published 17 September 2004)

\begin{abstract}
The magnetic transition in a FeSiBCuNb nanocrystalline alloy, associated with the decoupling of ferromagnetic crystallites around the Curie point of the residual amorphous matrix, is analyzed in this work through the temperature dependence of the ac axial magnetic permeability and impedance of the samples. The temperature dependence of both complex magnitudes presents a maximum in the irreversible contribution at a certain transition temperature. While for low values of the exciting ac magnetic field the transition temperature lies below the Curie temperature of the amorphous phase, a shift above this Curie point is observed increasing the amplitude of the applied ac magnetic field. The detected field dependence is interpreted taking into account the ac nature of the inductive characterization techniques and the actual temperature dependence of the coercivity of the samples.
\end{abstract}

DOI: 10.1103/PhysRevB.70.094412

\section{INTRODUCTION}

The magnetic properties of nanocrystalline materials composed of crystals of a nanometer-range surrounded by a residual amorphous matrix has been extensively studied during the last decade. ${ }^{1}$ In these nanocrystalline materials the magnetic interactions between the two constituent magnetic phases mainly determine the macroscopic magnetic behavior and its temperature dependence. In this sense, the observed magnetic transition around the Curie point of the residual amorphous phase is associated with a magnetic decoupling between the ferromagnetic crystallites with higher Curie point. ${ }^{2}$ Within the framework of the random anisotropy model the occurrence of this magnetic transition is basically ascribed to a decrease in the effective exchange constant around the Curie point of the residual amorphous phase, $T_{C 2}$, leading to a sharp increase in the effective magnetocrystalline constant. ${ }^{3,4}$ Accordingly, the magnetic transition is experimentally detected as a sharp increase and decrease, respectively, of the coercivity ${ }^{5}$ and magnetic permeability ${ }^{6}$ around $T_{C 2}$.

The magnetic properties of these two-phase systems, in particular, the temperature dependence of their soft magnetic behavior, represents a research topic of continuous interest from both basic and applied point of views. As an example, the shift of the magnetic transition (magnetic decoupling between ferromagnetic crystallites) above $T_{C 2}$ has been extensively reported and correlated to the occurrence of exchange coupling between the ferromagnetic grains through the paramagnetic amorphous matrix. ${ }^{7,8}$ However, there are some experimental facts related with the analysis of the magnetic transition and the magnetic decoupling of ferromagnetic crystallites that needs to be analyzed in further detail. In particular, when this magnetic transition is analyzed through the temperature dependence of the ac magnetic permeability,
PACS number(s): 75.50.Tt, 75.50.Bb, 75.30.Cr, 75.30.Et

a maximum in the irreversible contribution is detected at measuring temperature, $T_{P}$, around $T_{C 2}$. Surprisingly, a shift in $T_{P}$ towards higher measuring temperatures is experimentally observed with the amplitude of the exciting magnetic field. ${ }^{9}$

Our aim in the present work is to further analyze the characteristic magnetic transition in a soft nanocrystalline $\mathrm{FeSiBCuNb}$ alloy around the Curie point of the residual amorphous phase by means of ac inductive techniques, that is, the ac axial magnetic permeability and the electrical impedance. While the ac magnetic permeability is a well known characterization technique widely employed in the characterization of different magnetic transitions (i.e., freezing temperature in spin glasses,${ }^{10}$ martensitic transformation in magnetic memory shape alloys, ${ }^{11}$ spin reorientation transition in rare earths ${ }^{12}$ ), the ac impedance has recently been proved as a very simple and versatile characterization technique. Basically, when an ac current flows through a soft magnetic sample, the associated transverse magnetic field magnetizes it, giving rise, for high enough current frequencies, to the so-called skin effect. In this high frequency region, where the current keeps mainly concentrated in a reduced region close to the sample surface, the complex impedance of the sample is mainly determined by the associated transverse magnetic permeability. ${ }^{13}$ Thus, the temperature dependence of the ac impedance can be directly employed to characterize those magnetic transitions associated with noticeable changes in the magnetic permeability of the samples: metal-insulator transition in manganites, ${ }^{14}$ Curie point in Heusler alloys, ${ }^{15}$ spin reorientation in $\mathrm{Gd},{ }^{16}$ the martensitic transformation in magnetic memory shape alloys ${ }^{16}$ and the magnetic decoupling of the ferromagnetic crystallites in soft nanocrystalline samples around $T_{C 2} \cdot{ }^{17,18}$

In the present work, the temperature dependence of the ac axial magnetic permeability, $\mu=\mu_{\mathrm{r}}+i \mu_{\mathrm{i}}$, and impedance, 


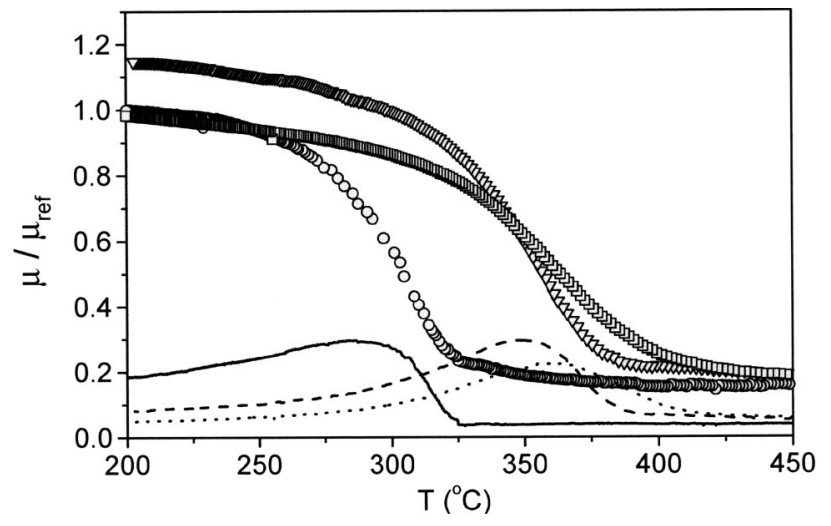

FIG. 1. Temperature $(T)$ dependence of the real, $\mu_{r}$, (symbols) and imaginary, $\mu_{i}$, (lines) components of the magnetic permeability, $\mu=\mu_{r}+i \mu_{i}$ of the nanocrystalline $\mathrm{Fe}_{73.5} \mathrm{Si}_{13.5} \mathrm{~B}_{9} \mathrm{Cu}_{1} \mathrm{Nb}_{3}$ alloy $\left(t_{\text {ann }}\right.$ $=60 \mathrm{~min})$ for different values of the amplitude of the exciting magnetic field, $H_{a c}(f=500 \mathrm{~Hz})(\bigcirc)$ and $(-)$ for $H_{a c}=9.6 \mathrm{~A} / \mathrm{m} ;(\nabla)$ and (---) for $H_{a c}=48 \mathrm{~A} / \mathrm{m} ;(\square)$ and $(\cdots)$ for $H_{a c}=62.5 \mathrm{~A} / \mathrm{m}$.

$Z=R+\mathrm{i} X$, is analyzed in ribbon and wire shape nanocrystalline $\mathrm{FeSiBCuNb}$ alloys. Both complex magnitudes present a maximum value in the irreversible contribution at a certain transition temperature, $T_{P}$. The detected field dependence of $T_{P}$ (i.e., an increase in $T_{P}$ with the amplitude of the exciting ac field acting on the sample) is interpreted in terms of the ac nature of the inductive characterization techniques and the actual temperature dependence of the coercivity of the samples.

\section{EXPERIMENTAL PROCEDURE}

The samples, amorphous ribbons $(\approx 1 \mathrm{~mm}$ wide and $20 \mu \mathrm{m}$ thick) and wires (diameter $\approx 120 \mu \mathrm{m}$ ) with nominal compositions $\mathrm{Fe}_{73.5} \mathrm{Si}_{13.5} \mathrm{~B}_{9} \mathrm{Cu}_{1} \mathrm{Nb}_{3}$ were obtained by rapid quenching from the melt (melt spinning and in-rotatingwater-quenching techniques, respectively). The desired nanocrystalline structure was obtained through isothermal treatments in vacuum atmosphere at $550{ }^{\circ} \mathrm{C}$. The temperature dependence of the ac axial magnetic permeability and impedance was characterized by a conventional induction method and the four-probe technique, respectively, in a temperature range from 200 to $450{ }^{\circ} \mathrm{C}$. The in-phase and outof-phase voltage components with respect to the exciting magnetic field (along the axial and transverse directions for the axial magnetic permeability and impedance measurements, respectively) were suitably detected with the help of a lockin-amplifier.

\section{RESULTS}

\section{A. Temperature dependence of the magnetic permeability}

Figure 1 shows the temperature dependence of the magnetic permeability, $\mu=\mu_{\mathrm{r}}+i \mu_{\mathrm{i}}$, for the $\mathrm{Fe}_{73.5} \mathrm{Si}_{13.5} \mathrm{~B}_{9} \mathrm{Cu}_{1} \mathrm{Nb}_{3}$ ribbon $\left(5 \mathrm{~cm}\right.$ in length) annealed at $550{ }^{\circ} \mathrm{C}$ during $60 \mathrm{~min}$ for different values of the amplitude of the applied axial ac field, $H_{\mathrm{ac}}=9.6,48$ and $62.5 \mathrm{~A} / \mathrm{m}$ (frequency, $f=500 \mathrm{~Hz}$ ). The displayed permeability values are normalized to the

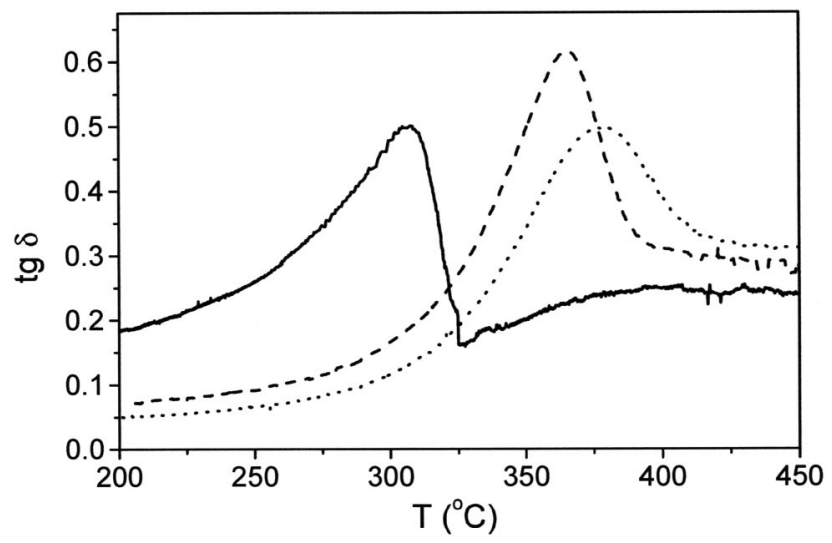

FIG. 2. Loss factor, $\tan \delta$, versus temperature, $T$, for the nanocrystalline $\mathrm{Fe}_{73.5} \mathrm{Si}_{13.5} \mathrm{~B}_{9} \mathrm{Cu}_{1} \mathrm{Nb}_{3}$ alloy $\left(t_{\text {ann }}=60 \mathrm{~min}\right)$ for different values of the amplitude of the exciting ac magnetic field, $H_{a c}(f$ $=500 \mathrm{~Hz}):(-) 9.6 \mathrm{~A} / \mathrm{m}, \quad(--) 48 \mathrm{~A} / \mathrm{m}$ and $(\cdots)$ for $H_{a c}$ $=62.5 \mathrm{~A} / \mathrm{m}$.

value of the real component at $200{ }^{\circ} \mathrm{C}$ for $H_{a c}=9.6 \mathrm{~A} / \mathrm{m}$ $\left[\mu_{\mathrm{ref}}=\mu_{\mathrm{r}}\left(200{ }^{\circ} \mathrm{C}, H_{\mathrm{ac}}=9.6 \mathrm{~A} / \mathrm{m}\right)\right]$. As Fig. 1 shows, the temperature dependence of the real $\left(\mu_{\mathrm{r}}\right)$ and imaginary $\left(\mu_{\mathrm{i}}\right)$ permeability components are characterized by a sharp decrease and a maximum value, respectively, around a certain transition temperature. This maximum in $\mu_{\mathrm{i}}$ is a characteristic feature of different magnetic transitions (i.e., freezing temperature in spin glasses, ${ }^{19}$ blocking temperature of superparamagnetic particles ${ }^{20}$ ). In the present case, the occurrence of such a maximum could be ascribed to the magnetic decoupling between ferromagnetic crystallites for temperatures close to the Curie temperature of the residual amorphous phase, $T_{C 2}\left(T_{C 2} \approx 340{ }^{\circ} \mathrm{C}\right.$ estimated from previous thermogravimetry studies, that is, the temperature dependence of dc saturation magnetization, $M_{S}(T)$. $)^{21}$ Note that the magnetic transition surprisingly shifts towards higher temperatures with the amplitude of the ac magnetic field, $H_{\mathrm{ac}}$.

The temperature dependence of the loss factor, $\tan \delta$ $=\mu_{i} / \mu_{r}$, related to the irreversible contribution of the magnetization process, enables the characterization of the magnetic transition in these nanocrystalline samples. ${ }^{22}$ Since $\tan \delta$ is associated with the energy loss per cycle, it should display a similar temperature dependence than the coercive field of the samples, that is, a maximum value at $T=T_{P}$ associated with the uncoupled state of the single domain ferromagnetic particles. ${ }^{5}$ Figure 2 shows the temperature dependence of $\tan \delta$ for the different values of $H_{\mathrm{ac}}$. Notice the increase of $T_{P}$ (close to $100{ }^{\circ} \mathrm{C}$ ) with the amplitude of the ac magnetic field.

In order to analyze the influence of the exciting frequency, $f$, in the observed shift of $T_{P}$, Fig. 3 displays the temperature evolution of $\tan \delta$ for $f=200,500$ and $1000 \mathrm{~Hz}$ and $H_{\mathrm{ac}}$ $=6.9$ and $63.5 \mathrm{~A} / \mathrm{m}$. First, the increase of $f$ gives rise, irrespectively of the amplitude of the exciting ac field to an overall increase in the values of $\tan \delta$ compatible with the associated increase in the energy losses per cycle (eddycurrent losses). At constant $H_{a c}$, the position of maximum does not noticeably change, except for a slight shift towards lower temperatures with $f$ [also detected in $\mu_{i}(f)$ ]. Thus, the 


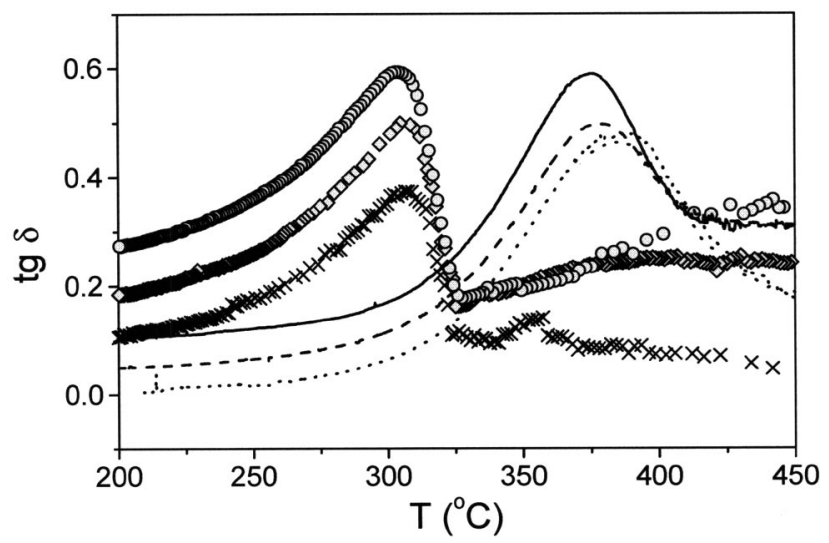

FIG. 3. Temperature evolution of the loss factor, $\tan \delta$, at different values of the ac magnetic field (symbols: $H_{a c}=9.6 \mathrm{~A} / \mathrm{m}$; lines: $\left.H_{a c}=62.5 \mathrm{~A} / \mathrm{m}\right)$ and exciting frequency: $(\times)$ and $(\cdots)$ for $f$ $=200 \mathrm{~Hz} ;(\diamond)$ and $(--)$ for $f=500 \mathrm{~Hz} ;(\bigcirc)$ and $(-)$ for $f=1 \mathrm{kHz}$.

detected increase in $T_{P}$ with the amplitude of the exciting ac field, should be correlated with some basic characteristics of the magnetic transition around $T_{C 2}$, independent of the dynamical characteristics of the decoupling process. In fact, if the maximum in the irreversible contribution is interpreted within the classical models associated with relaxation processes (Debye theory) a different $T_{P}(f)$ should be found (i.e., an increase of $T_{P}$ with $f$ in thermally activated fine particles systems). ${ }^{23}$ Therefore, in the present case, the detected decrease of $T_{P}$ with $f$ would have basically an inductive origin associated with the eddy-current contribution in the metallic sample (decrease with $f$ of the effective magnetic field acting on the sample).

To further check whether this shift with $H_{a c}$ is a specific feature of the analyzed magnetic transition (magnetic decoupling of the ferromagnetic crystallites), the amorphous alloy was analyzed under similar experimental conditions. A previous annealing at $360{ }^{\circ} \mathrm{C}$ for $10 \mathrm{~min}$ was performed to stabilize the amorphous sample. Such an annealing gives rise to a relaxation of the amorphous structure without any further structural modification. As Fig. 4 shows, where the temperature evolution of both permeability components are shown for $H_{a c}=6.9$ and $48 \mathrm{~A} / \mathrm{m}(f=500 \mathrm{~Hz})$, the magnetic transition (Curie point, $T_{C 2}$ ) takes place at $T \approx T_{C 2}=340{ }^{\circ} \mathrm{C}$ irrespectively of the amplitude of the applied field. It is also remarkable that in this amorphous state the imaginary component $\mu_{i}$ does not present any noticeable maximum around $T_{C 2}$.

\section{B. Temperature dependence of the impedance}

To clarify the origin of the detected field dependence of $T_{P}$, the magnetic transition was characterized through the temperature dependence of the ac impedance (external magnetic field $H=0)$. In order to enhance the magnetoinductive contribution (increase of the transverse magnetic permeability) a wire shaped nanocrystalline alloy was selected for the impedance measurements. A piece $5 \mathrm{~cm}$ in length ( $\mathrm{Fe}_{73.5} \mathrm{Si}_{13.5} \mathrm{~B}_{9} \mathrm{Cu}_{1} \mathrm{Nb}_{3}$ nominal composition) was submitted to subsequent annealings $\left(T_{\mathrm{ann}}=550^{\circ} \mathrm{C}\right)$ during 5,10 and

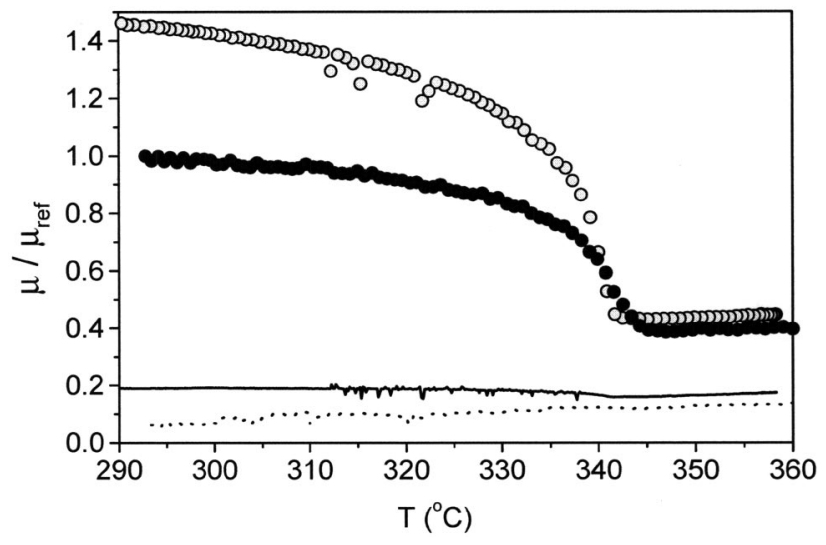

FIG. 4. Temperature dependence of the real, $\mu_{r}$ (symbols) and imaginary, $\mu_{i}$ (lines) components of the magnetic permeability, $\mu$ $=\mu_{r}+i \mu_{i}$, of the relaxed amorphous $\mathrm{Fe}_{73.5} \mathrm{Si}_{13.5} \mathrm{~B}_{9} \mathrm{Cu}_{1} \mathrm{Nb}_{3}$ alloy for different values of the amplitude of the exciting magnetic field $(f$ $=500 \mathrm{~Hz})$ : (๑) and (--) $H_{a c}=9.6 \mathrm{~A} / \mathrm{m} ;$ (O) and (-) $H_{a c}$ $=48 \mathrm{~A} / \mathrm{m}$.

$30 \mathrm{~min}$ ( $T 1$ and $T 2$ and $T 3$ nanocrystalline states, respectively). Figures 5(a) and 5(b) show the temperature dependence of the complex impedance components, $Z=R+\mathrm{i} X$, for
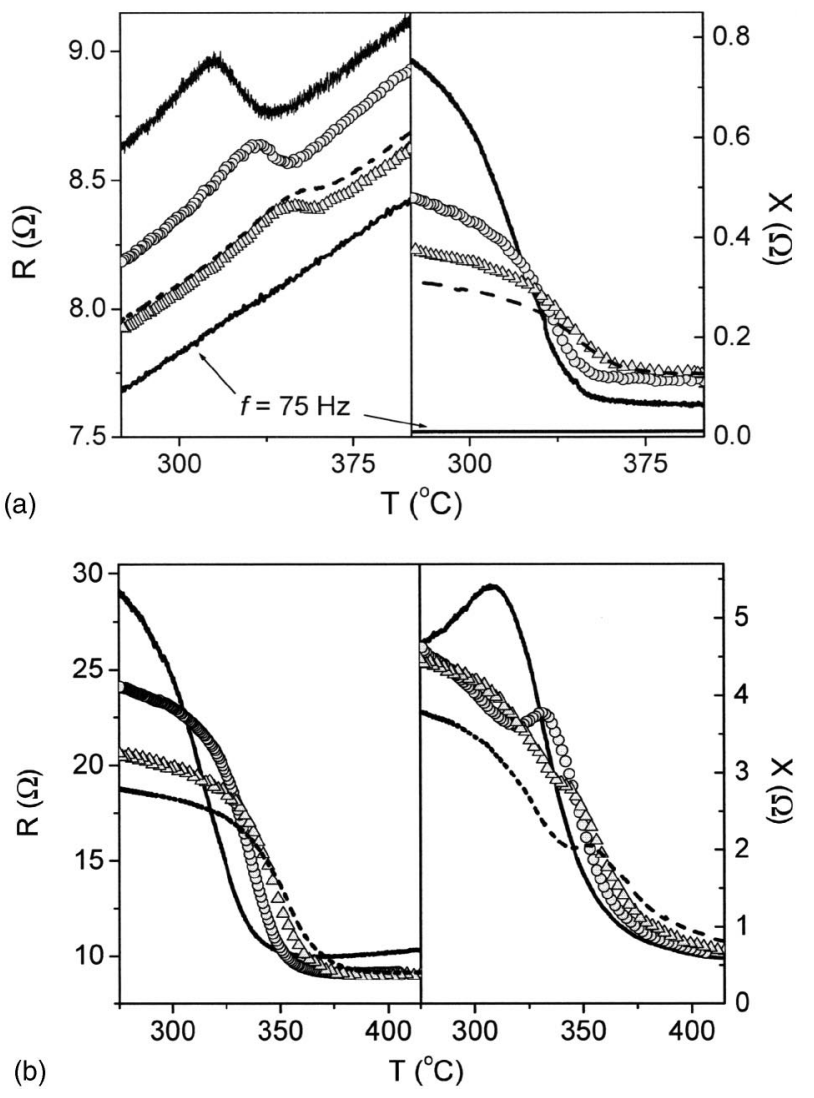

FIG. 5. Temperature dependence of the impedance components, $Z=R+\mathrm{i} X$, for (a) $f=1 \mathrm{kHz}$ and (b) $f=100 \mathrm{kHz}$ for the $T 3$ nanocrystalline wire as a function of the amplitude of the exciting current, $I_{a c}:(-) 5 \mathrm{~mA},(\bigcirc) 10 \mathrm{~mA},(\triangle) 15 \mathrm{~mA}$ and (--) $20 \mathrm{~mA}$. [The impedance data for $f=75 \mathrm{~Hz}$ and $I_{a c}=5 \mathrm{~mA}$ are included in Fig. 5(a)]. 
$f=1$ and $100 \mathrm{kHz}$, respectively, at different values of the amplitude of the exciting ac current, $I_{a c}$, for the final $T_{3}$ nanocrystalline sample. For comparison, Fig. 5(a) also shows the temperature dependence of the impedance components at $f=75 \mathrm{~Hz}$.

First, at low frequency [see Fig. 5(a)], the impedance components directly reflect the inductive contribution associated with the temperature dependence of the circumferential magnetic permeability, $\mu_{\phi}$. In this low frequency region, the sample voltage can be expressed as ${ }^{24}$

$$
V=R_{d c} I+i\left\langle E_{Z}\right\rangle L,
$$

where $R_{d c}$ and $L$ are the Ohmic resistance and the sample length, respectively, and $\left\langle E_{Z}\right\rangle$ is the average electrical field originated by the circumferential magnetization process $\left(\left\langle E_{Z}\right\rangle \propto \mu_{\phi}\right)$. Thus, taking into account the complex nature of the circumferential permeability $\left(\mu_{\phi}=\mu_{\phi, r}+\mathrm{i} \mu_{\phi, i}\right)$, the occurrence of the maximum value in $R$ and the sharp decrease in $X$ around $T_{C 2}$ basically reflect the main characteristics of temperature dependence of the imaginary and real components of $\mu_{\phi}$, respectively. Notice the clear shift of the magnetic transition with $I_{a c}$. This behavior reflects the dependence of $\mu_{\phi}(T)$ on the mean circumferential magnetic field $\left(H_{\phi} \propto I_{a c}\right)$ acting on the sample.

With respect to the impedance response at $f=100 \mathrm{kHz}$, the increase of the exciting frequency gives rise in the coupled state (low temperature) to a clear increase in both impedance values [see Fig. 5(b)]. In this range of high frequency values, where the so-called skin effect takes place, the electrical current is mainly concentrated in the sample region close to the wire surface. According to the classical electrodynamical model, ${ }^{25}$ the complex impedance, $Z$, is directly expressed in terms of the Bessel functions of the first kind $J_{i}(k a), Z=\frac{1}{2} R_{d c} k a J_{0}(k a) / J_{1}(k a)$, with $a$ : wire radius and $k=\sqrt{i 2 \pi \mu_{\phi} f / \rho}$. The decrease of $\mu_{\phi}$ associated with the magnetic decoupling of the ferromagnetic crystallites for $T>T_{C 2}$, has associated, as Fig. 5(b) shows, a drastic decrease in both impedance components. Again, as in the previous cases, the increase of the exciting magnetic field (increase in $I_{a c}$ ) gives rise to a clear displacement of the magnetic transition towards higher measuring temperatures.

\section{DISCUSSION}

The basic magnetic characteristics of these biphasic nanocrystalline materials can be suitably described within the framework of the random anisotropy models. ${ }^{1,26}$ In particular, in the coupled state where the magnetocrystalline anisotropy of the ferromagnetic crystallites is averaged out via the magnetic interactions between both constituent magnetic phases, the initial magnetic permeability can be expressed as

$$
\mu=p_{\mu} \frac{J_{S}^{2}}{\mu_{0} k_{\mathrm{eff}}},
$$

with $p_{\mu}$ a dimensionless factor; $J_{S}=\nu_{c} J_{1}+\left(1-\nu_{c}\right) J_{2}$, with $J_{i}$ $=\mu_{0} M_{S i}, M_{S i}$ : saturation magnetization with $i=1$ and 2 for the crystalline and residual amorphous phases, respectively; $\nu_{c}$ : crystalline volume fraction; and $k_{\text {eff }}$ the effective magnetocrystalline anisotropy defined as

$$
k_{\mathrm{eff}}=\frac{\nu_{c}^{2} k_{l}^{4} d^{6}}{A^{3}}
$$

with $k_{1}$ and $d$ the magnetocrystalline anisotropy constant and mean grain size, respectively, of the crystalline phase and $A$ the mean exchange constant of the coupled system, $A$ $=\left(A_{1} A_{2}\right)^{1 / 2}\left(A_{1}\right.$ and $A_{2}$ the mean exchange constants of the crystalline and amorphous phases, respectively). For $T$ $\leqslant T_{C 2}$ and assuming $k_{1}$ and $A_{1}$ roughly constants, $k_{\text {eff }}(T)$ would be mainly governed by the temperature dependence of $A_{2}$. Thus, the decrease of $A_{2}(T)$ around the Curie point of the residual amorphous phase has associated a magnetic hardening correlated with the parallel increase in $k_{\text {eff }}(T)$ [see Eq. (3)]. Thus, for $T \approx T_{C 2}$ the effective magnetic anisotropy reaches a maximum value close to $k_{1}$ and the magnetic decoupling between the ferromagnetic grains takes place.

\section{A. Enhancement of the exchange coupling with the applied magnetic field}

The displacement of the magnetic transition (decoupling of the ferromagnetic crystallites) above $T_{C 2}$ in nanocrystalline soft magnetic samples has been previously reported, ${ }^{7,8}$ mainly through the analysis of the temperature dependence of the coercivity, $H_{c}$. In those reports, the shift is mainly observed in samples with high crystalline fractions $\left(\nu_{c} \approx 1\right)$ where the mean distance between the ferromagnetic grains (width of the paramagnetic amorphous phase) is within the nanometer range $(\approx 1 \mathrm{~nm})$. Its origin was generally ascribed to the exchange coupling between ferromagnetic grains through the paramagnetic amorphous matrix,,${ }^{27,28}$ in a similar way as the widely reported effect in multilayered systems ${ }^{29}$ (indirect or RKKY exchange interaction). In fact, Monte Carlo simulations have been recently employed to explain the enhancement of the Curie temperature of the residual amorphous phase in terms of the magnetic polarization of the matrix by exchange fields arising from the nanocrystalline grains. ${ }^{30}$

Therefore, considering that $T_{P}$ (measuring temperature where $\tan \delta$ reaches a maximum value) represents the actual temperature ascribed to the exchange decoupling of the ferromagnetic crystallites, the field dependence of $T_{P}$ could be explained according to the previous discussion, with a parallel increase of $T_{C 2}$ with $H$. However, this assumption would imply according to the present experimental results an increase of $T_{C 2}$ close to $100 \mathrm{~K}$, incompatible with previous dc thermogravimetry measurements in the alloy ${ }^{21}$ (maximum dc applied magnetic field $\approx 4 \mathrm{kA} / \mathrm{m}$ ) and with the reported $T_{C 2}$ values in similar compositions. ${ }^{1}$ Therefore, assuming $T_{C 2}$ roughly constant with $H$, the detected changes in $T_{P}$ can be interpreted to be a consequence of the reinforcement of the exchange coupling of the ferromagnetic crystallites with the applied magnetic field. Thus, the occurrence of exchange coupling above $T_{C 2}$ and its reinforcement with $H$, would be associated according to Eq. (3) to a parallel increase of the mean exchange constant of the paramagnetic residual amorphous phase, $A_{2}$, i.e., magnetization of the paramagnetic phase under the action of the effective molecular field generated by the remainder ferromagnetic phase. ${ }^{31}$ This simple 


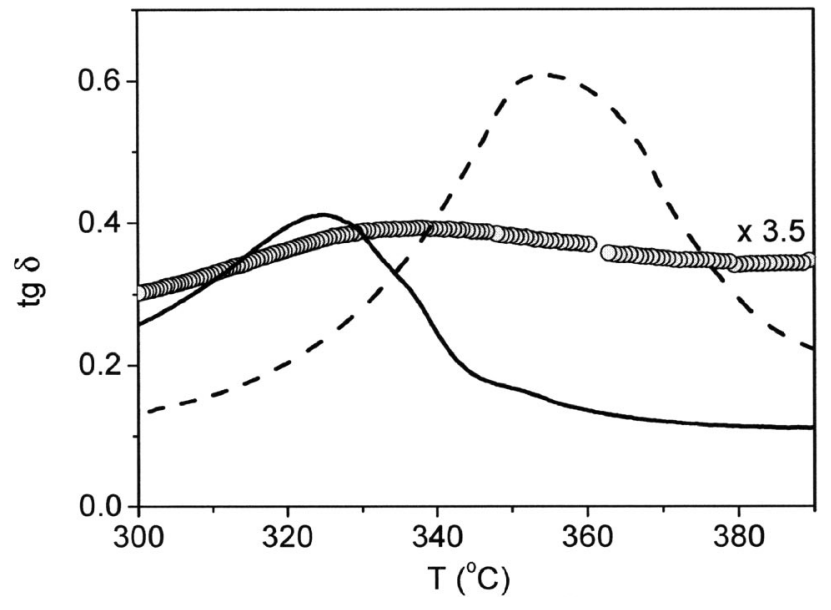

FIG. 6. Temperature dependence of the loss factor, $\tan \delta$, at $f$ $=500 \mathrm{~Hz}$ for the $T 2$ nanocrystalline wire at $H_{a c}=38 \mathrm{~A} / \mathrm{m}$ (solid line), $H_{a c}=152 \mathrm{~A} / \mathrm{m}$ (dashed line) and $H_{a c}=38 \mathrm{~A} / \mathrm{m}$ plus $H_{d c}$ $=150 \mathrm{~A} / \mathrm{m}$ (symbol).

assumption would explain the main experimental facts obtained in the present study, that is, the increase of the transition temperature with the applied magnetic field for $T>T_{C 2}$ as a consequence of the parallel increase in the mean effective molecular field (alignment of the ferromagnetic crystallites).

However, we must keep in mind the alternative (ac) nature of the magnetic field employed in the present magnetic characterization. If the main origin of the detected field dependence of the magnetic transition is correlated with an enhancement of the mean exchange constant, the magnetic field effect should be independent of its alternative (ac) or constant $(\mathrm{dc})$ characteristics. Figure 6 displays the temperature dependence of $\tan \delta(f=500 \mathrm{~Hz})$ for the nanocrystalline wire (intermediate $T 2$ annealed state) at $H_{a c}=38 \mathrm{~A} / \mathrm{m}$ (solid line) and under a bias dc field $H_{d c}=150 \mathrm{~A} / \mathrm{m}$ (symbols). For comparison, $\tan \delta(T)$ for $H_{a c}=152 \mathrm{~A} / \mathrm{m}\left(H_{d c}=0\right)$ is also included (dashed line). First, the application of the dc field gives rise to a clear decrease in $\tan \delta$ as a direct consequence of the decrease in the irreversible component of the ac magnetic permeability (approach to saturation). However, the application of the bias dc field just surprisingly promotes a slight increase in the defined transition temperature, $T_{P}$, in comparison with the detected displacement for $H_{a c}$ $=152 \mathrm{~A} / \mathrm{m}$. Therefore, although the occurrence of some enhancement of the exchange interaction with $H_{\mathrm{dc}}$ cannot be completely excluded, the different response of $\tan \delta(T)$ under ac or dc magnetic fields, points to a different origin of the detected field dependence of $T_{P}$.

\section{B. Temperature dependence of coercivity and correlation with the field dependence of the magnetic permeability}

Figures 7(a) and 7(b) show the temperature dependence of the axial coercive field, $H_{c}$, (obtained through the induction method at $f=100 \mathrm{~Hz}$ ) and the transition temperature, $T_{P}$ (defined from the maximum in $\tan \delta$ at $f=500 \mathrm{~Hz}$ ) versus $H_{\text {ac }}$ in the three annealed states $(T 1, T 2$ and $T 3)$ of the nanocrystal-

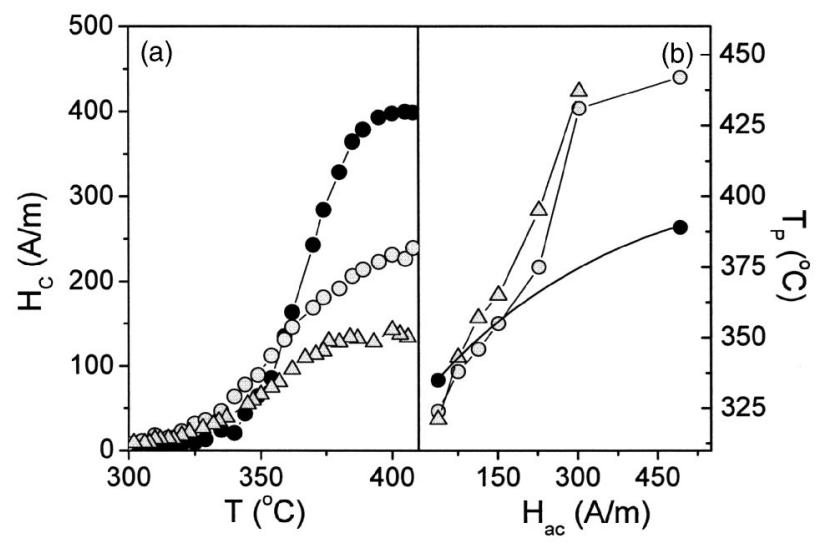

FIG. 7. (a) Coercive field, $H_{C}$, versus measuring temperature, $T$, for $(\bigcirc) T 1,(\bigcirc) T 2$ and $(\triangle) T 3$ annealed states of the nanocrystalline wire. (b) Transition temperature, $T_{P}$, as a function of the amplitude of the exciting ac magnetic field, $H_{a c}$, for $(\bigcirc) T 1$, (○) $T 2$ and $(\triangle) T 3$ annealed states of the nanocrystalline wire.

line wire. As can be observed, there is an inverse correlation between $H_{c}$ and $T_{P}$, that is, the sample with the smoother increase in $H_{c}(T)$ and thus the lowest coercivity in the uncoupled state (T3 annealed state) displays the strongest field dependence of $T_{P}$ (highest $T_{P}$ values). This behavior can be well understood taking into account the ac nature of the magnetic field employed in the $T_{P}$ characterization. Consider an amplitude of the exciting ac magnetic field higher than $H_{C}$ at low measuring temperatures (i.e., $H_{\mathrm{ac}}=100 \mathrm{~A} / \mathrm{m}$ ). As $T$ increases, the progressive magnetic hardening around $T_{C 2}$ (magnetic decoupling of the ferromagnetic crystallites) promotes a parallel decrease and increase, respectively, in $\mu_{r}(T)$ and $\mu_{i}(T)$ (see for instance Fig. 1). However, at a certain measuring temperature, $T^{*}$, the amplitude of the ac magnetic field equals the coercivity of the sample $\left(H_{\mathrm{ac}}=H_{C}\right)$. A further increase of $T$ above this critical point leads to a parallel decrease in both permeability components since the applied magnetic field is not able to magnetize the assembly of ferromagnetic single particles $\left(H_{\mathrm{ac}}<H_{C}\right)$. Thus, the detected temperature dependence of both permeability components can be easily explained in terms of the relationship between $H_{\mathrm{ac}}$ and $H_{C}(T)$, that is, a continuous decrease of $\mu_{r}$ around $T_{C 2}$ and a maximum value in $\mu_{i}$ at the measuring temperature $T^{*}$ where $H_{\mathrm{ac}}=H_{C}$.

The temperature and field evolution of both permeability components mainly determine the impedance response of the samples. As Fig. 5 shows, the increase of the amplitude of the ac exciting current, $I_{\text {ac }}$ (increase in the mean ac circumferential field, $H_{\phi}$ ), promotes a similar shift in the temperature dependence of both impedance components. Thus, the detected maximum in the imaginary circumferential permeability (maximum in the resistive component at low frequency, $f=1 \mathrm{kHz}$ ) would correspond with the measuring temperature where the mean amplitude of $H_{\phi}$ equals the circumferential coercivity of the sample. For higher exciting frequencies $(f=100 \mathrm{kHz})$ where the skin effect takes place, both impedance components drastically decrease at this measuring temperature (see Fig. 5). Above this critical temperature, the mean circumferential field is not able to magnetize 


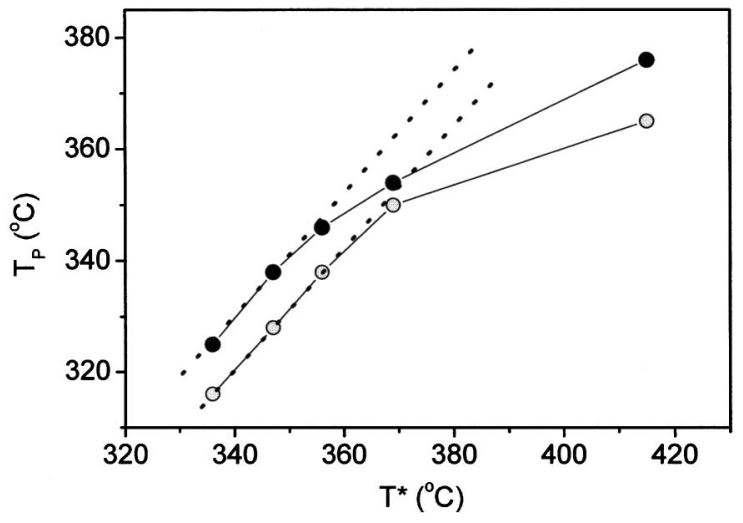

FIG. 8. Transition temperature, $T_{\mathrm{P}}$, defined from the maximum in $(\bigcirc)$ the loss factor, $\tan \delta$, or $(\bullet)$ the imaginary component of the magnetic permeability, $\mu_{i}$, versus the measuring temperature $T^{*}\left(H_{a c}=H_{C}\right)$. The dotted line in the figure represents the linear fit with slope 1 .

the assembly of single domain particles, leading to a sharp increase in the characteristic magnetic penetration depth, and thus to the disappearance of the skin effect of the sample.

According to the previous discussion, a direct relationship between the defined apparent transition temperature, $T_{P}$ (maximum in $\tan \delta$ ) and $T^{*}$ (temperature where $H_{\text {ac }}$ equals $\left.H_{C}\right)$ should be found. Figure 8 shows $T_{P}$ versus $T^{*}$ for the intermediate $T 2$ annealed state. For comparison, the temperature associated with the maximum value in the imaginary component of the axial permeability, $\mu_{i}$, is also shown (open circles). As can be observed, there is a direct correlation between both temperatures. In particular, a linear dependence (the dotted line in Fig. 8 represents the linear fit with slope 1) is directly found at low temperatures (low amplitude $H_{\mathrm{ac}}$ ).

In conclusion, the main experimental facts of the present study can be summarized as follows.

(i) The occurrence of a maximum in the irreversible contribution $\left(\tan \delta\right.$ or $\mu_{i}$ ) at $T_{P} \approx T^{*}$ where the amplitude of the exciting ac magnetic field equals the sample coercivity $\left(H_{\mathrm{ac}}=H_{C}\right)$.

(ii) The increase of $T_{P}$ with $H_{\mathrm{ac}}$ as a result of the increase of $H_{C}$ with $T$ (magnetic decoupling of the ferromagnetic crystallites around $T_{C 2}$ ), that is, the condition $H_{\mathrm{ac}}=H_{C}$ is fulfilled at higher temperatures as $H_{\mathrm{ac}}$ increases.

(iii) Those samples with the smoothest increase in $H_{C}(T)$ (highest crystalline fraction, $T 3$ in the present study) show a strongest field dependence of $T_{P}$ (highest $T_{P}$ values).

\section{CONCLUSIONS}

The magnetic transition associated with the magnetic decoupling between ferromagnetic crystallites around the Curie point of the residual amorphous phase, $T_{C 2}$, is analyzed in a $\mathrm{Fe}_{73.5} \mathrm{Si}_{13.5} \mathrm{~B}_{9} \mathrm{CuNb}_{3}$ alloy (ribbon and wire). In particular, two ac inductive techniques and their temperature dependence are employed in this study: axial magnetic permeability and electrical impedance. With respect to the permeability characterization, a maximum value in the loss factor, $\tan \delta$, is experimentally detected at a certain measuring temperature (transition temperature, $T_{P}$ ). While for a low value of the exciting ac magnetic field, $H_{\mathrm{ac}}$, the transition temperature lies below $T_{C 2}$, a shift above this Curie point is experimentally detected as $H_{\mathrm{ac}}$ increases. Similar results are obtained through the impedance characterization, both in the low frequency region (magnetoinductance effect) and at high frequency $(100 \mathrm{kHz})$ where the skin effect dominates the impedance response of the sample. In this case, the shift of the apparent magnetic transition takes place with the increase in the amplitude of the exciting current (an increase in the mean ac circumferential field acting on the sample).

The origin of the detected field dependence is first analyzed in terms of the reinforcement of the exchange coupling between ferromagnetic crystallites with the applied magnetic field. However, although the occurrence of some exchange field enhancement cannot be completely excluded, the main experimental facts of the field dependence of $T_{P}$ and its evolution with the thermal treatments are suitably explained taking into account the ac nature of the performed magnetic characterization. The results indicate a direct relationship between the defined transition temperature and the measuring temperature where the amplitude of the ac magnetic field equals the sample coercivity $\left(H_{\mathrm{ac}}=H_{C}\right)$. Thus, the detected increase of $T_{P}$ with $H_{\text {ac }}$ results as a direct consequence of the characteristic increase in $H_{C}$ ascribed to the exchange decoupling process of the ferromagnetic crystallites above the $\mathrm{Cu}-$ rie point of the residual amorphous phase.

Finally, the versatility should be remarked, but mainly, the simplicity of the ac impedance as a characterization technique in the analysis of those magnetic transitions associated with noticeable changes in the magnetic permeability.

\section{ACKNOWLEDGMENTS}

This work was supported by the Spanish Gobierno de Navarra under project "Propiedades de magnetotransporte de nuevos materiales nanoestructurados."

\footnotetext{
*Electronic address: gpolo@unavarra.es

${ }^{1}$ G. Herzer, "Nanocrystalline soft magnetic alloys", in Handbook of Magnetic Materials, Edited by K. H. J. Buschow (Elsevier, Amsterdamm, 1997), Vol. 10, p. 415.

${ }^{2}$ A. Hernando, M. Vázquez, T. Kulik, and C. Prados, Phys. Rev. B 51, 3581 (1995).

${ }^{3}$ K. Suzuki and J. M. Cadogan, Phys. Rev. B 58, 2730 (1998).
}

${ }^{4}$ J. Arcas, A. Hernando, C. Gómez-Polo, F. J. Castaño, M. Vázquez, A. Neuweiler, and H. Krönmuller, J. Phys.: Condens. Matter 12, 3255 (2000).

${ }^{5}$ A. Hernando, P. Marín, M. Vázquez, J. M. Barandiarán, and G. Herzer, Phys. Rev. B 58, 366 (1998).

${ }^{6}$ Z. Wang, K. Y. He, J. Jim, J. He, L. Zhang, H. Zhang, and B. Shen, Mater. Sci. Eng., A 304-306, 1046 (2002). 
${ }^{7}$ A. Slawska-Waniewska, P. Nowicki, H. K. Lachowicz, P. Gorría, J. M. Barandiarán, and A. Hernando, Phys. Rev. B 50, 6465 (1994).

${ }^{8}$ A. Hernando and T. Kulik, Phys. Rev. B 49, 7064 (1994).

${ }^{9}$ C. Gómez-Polo, J. I. Pérez-Landazabal, and V. Recarte, IEEE Trans. Magn. 39, 3019 (2003).

${ }^{10}$ J. A. Mydosh, Spin Glasses: An Experimental Introduction (Taylor \& Francis, 1993), p. 64.

${ }^{11}$ O. Heczko, N. Lanska, O. Soderberg, and K. Ullakko, J. Magn. Magn. Mater. 242-245, 1446 (2002).

${ }^{12}$ J. M. D. Coey, K. Gallagher, and V. Skumryev, J. Appl. Phys. 87, 7028 (2000).

${ }^{13}$ M. Knobel, L. Kraus, and M. Vázquez, "Giant magnetoimpedance", in Handbook of Magnetism and Magnetic Materials, edited by K. H. J. Buschow (Elsevier, New York, 2003), Vol. 12, p. 497.

${ }^{14}$ J. Hu and H. Qin, Mater. Sci. Eng., B 100, 304 (2003).

${ }^{15}$ G. L. F. Fraga, P. Pureur, and D. E. Brandao, Solid State Commun. 124, 7 (2002).

${ }^{16}$ V. V. Khovailo, T. Abe, and T. Takagi, J. Appl. Phys. 94, 2491 (2003).

${ }^{17}$ L. Zeng, Z. J. Zhao, X. L. Yanga, J. Z. Ruan, and G. Chen, J. Magn. Magn. Mater. 246, 422 (2002).

${ }^{18}$ C. Gómez-Polo and M. Vázquez, J. Magn. Magn. Mater. 272276, 1853 (2004).

${ }^{19}$ D. Hüser, L. E. Wenger, A. J. van Duyneveldt, and J. A. Mydosh, Phys. Rev. B 27, 3100 (1983).
${ }^{20}$ I. Skorvánek, S. Skwirblies and J. Kötzler, Phys. Rev. B 64, 184437 (2001)

${ }^{21}$ C. Gómez-Polo, P. Marín, L. Pascual, A. Hernando, and M. Vázquez, Phys. Rev. B 65, 024433 (2002).

${ }^{22}$ C. Gómez-Polo, J. I. Pérez-Landazabal, V. Recarte, J. Campo, P. Marín, M. López, A. Hernando, and M. Vázquez, Phys. Rev. B 66, 012401 (2002).

${ }^{23}$ P. C. Fannin, Studies of Magnetic Properties of Fine Particles and their Relevance to Materials Science (Elsevier, Amsterdam, 1992), p. 151.

${ }^{24}$ L. V. Panina, K. Mohri, K. Bushida, and M. Noda, J. Appl. Phys. 76, 6198 (1994).

${ }^{25}$ L. D. Landau, E. M. Lifschitz, and L. P. Pitaevski, Electrodynamics of Continuous Media (Buttherworth-Heinemann, London, 1995), p. 212.

${ }^{26}$ J. Arcas, A. Hernando, J. M. Barandiarán, C. Prados, M. Vázquez, P. Marín, and A. Neuweiler, Phys. Rev. B 58, 5193 (1998).

${ }^{27}$ A. Hernando, I. Navarro, and P. Gorría, Phys. Rev. B 51, 3281 (1995).

${ }^{28}$ J. S. Garitaonandia, D. S. Schmool, and J. M. Barandiarán, Phys. Rev. B 58, 12147 (1998).

${ }^{29}$ P. Günberg and D. Pierce, Encyclopedia of Materials: Science and Technology, (Elsevier North-Holland, Amsterdam 2001).

${ }^{30}$ O. Crisan, Y. Labaye, L. Berger, J. M. D. Coey, and J. M. Greneche, J. Appl. Phys. 91, 8727 (2002).

${ }^{31}$ A. González and A. Hernando, Phys. Rev. B 65, 094432 (2002). 